\title{
SARS-CoV-2 Lambda Variant: Spatiotemporal Distribution and Potential Public Health Impact
}

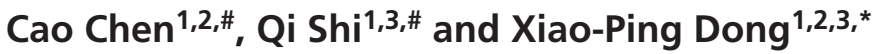

\begin{abstract}
Various SARS-CoV-2 variants have continually emerged since the summer of 2020. Recently, the spread and potential effects of the Lambda variant on public health have caused great scientific and public concern. The Lambda variant (C.37), first identified in Peru in December 2020, contains a novel deletion ( $\Delta 246-252)$ and two novel mutations, L452Q and F490S, not present in the ancestral strain and other variants. The Lambda variant was designated a variant of interest in April of 2021. By the end of July, this variant sequence was detected in more than 30 countries worldwide, mostly in South America. This study analyzed the global spatiotemporal distribution of the Lambda variant from the beginning of January to the end of July from publicly available data. The Lambda variant spread rapidly in Peru and became predominant in March. Circulation of the Lambda variant has also been observed in some neighboring countries, i.e., Argentina, Chile and Ecuador, where it has remained at remarkably low levels. The circulation of the Lambda variant in other countries in South America (e.g., Brazil and Colombia) and other regions of the world has also occurred at very low levels, even though this variant has been known for a long time. Multivariate linear regression analyses of the proportion of case fatalities attributable to the Lambda variant, the new deaths and the new confirmed cases per million (7-day rolling average) in Peru did not show significant associations. A review of the most recent data on the Lambda variant has suggested this variant's relatively high infectivity in cultured cells and low neutralizing titers of convalescent sera and vaccine-elicited antibodies in vitro. However, the exact effects of this variant on clinical severity and vaccine effectiveness remain poorly documented. The currently authorized COVID-19 vaccines are still believed to provide efficient protection against the Lambda variant.
\end{abstract}

Key words: SARS-CoV-2, COVID-19, the Lambda variant, spread, impact
Edited by:

Han Xia, Wuhan Institute of Virology, Chinese Academy of Science

Reviewed by:

Yanling Yang, China Agriculture University

Another reviewer requests to be anonymous

*Corresponding author:

E-mail: dongxp238@sina.com,

Fax: +86-10-58900815 (XPD)

${ }^{1}$ State Key Laboratory for Infectious Disease Prevention and Control, Collaborative Innovation Center for Diagnosis and Treatment of Infectious Diseases (Zhejiang University), National Institute for Viral Disease Control and Prevention, Chinese Center for Disease Control and Prevention, No. 155 Changbai Road, Beijing 102206, China

${ }^{2}$ Center for Biosafety Mega-Science, Chinese Academy of Sciences,

Wuhan City, Hubei Province 430071 , China

${ }^{3}$ China Academy of Chinese Medical Sciences, Beijing 100700, China

"The first two authors contributed equally to this article.

Received: August 182021

Revised: August 232021

Accepted: August 232021

Published Online: August 312021

\section{BACKGROUND}

The variation and evolution of SARSCoV-2, the etiological agent of COVID-19, has received substantial attention. Rapid progress in next-generation sequencing has enabled timely identification and recognition of SARS-CoV-2 variants. The WHO has classified variants into two main categories: variants of concern (VOCs) with evidence of significant effects on disease transmissibility, clinical severity and/or host immunity, and variants of interest (VOIs), with preliminary 
evidence, or evidence with major uncertainty, of effects on transmissibility, severity and/or immunity [1]. On this basis, the European Center for Disease Control and Prevention (ECDC) has used two additional categories [2]. One category is variants under monitoring, which may have similar properties to VOCs, but has evidence that is either weak or not yet assessed by the ECDC. The variants in this category must be present in at least one outbreak or detected in a community within the European Union/European Economic Area (EU/EEA), or must have evidence of community transmission of the variant elsewhere worldwide. the second category is de-escalated variants, which either have been circulating for a long time without affecting the overall epidemiological situation or are not associated with any concerning properties. The US Center for Disease Control and Prevention (USCDC) has also proposed variants of high consequence (VOHCs) in addition to VOCs and VOIs, for which preventive measures or medical countermeasures have significantly less effectiveness than those toward previously circulating variants [3]. As of August 20, 2021, four variants have been classified as VOCs. Among them, the Delta VOC is predominant worldwide and has shown strong effects on transmissibility, clinical severity and immune escape $[4,5]$. Four variants are VOIs, among which the Lambda variant has recently attracted substantial scientific and public attention. The other SARS-CoV-2 variants are undergoing further monitoring [1,2], and no VOHCs have been classified [3].

The Lambda variant was first detected in Peru in December 2020 and was designated a VOI on June 14, 2021.
It belongs to Pango lineage C.37. The Lambda variant has been highly concerning because of its apparent spread and possible association with increased numbers of fatal cases in some countries in South America. In this study, we analyzed the spatiotemporal distribution of the Lambda variant in the global context; comparatively evaluated the potential links between the spread of the Lambda variant and other epidemiological indexes; and reviewed the possible effects on public health according to the most recent studies.

\section{MUTATIONS OF THE LAMBDA VARIANT AFFECTING THE SPIKE PROTEIN}

Several point mutations and deletions involving amino acid substitutions have been well documented to affect the spike protein of the Lambda variant, as compared with the ancestral strain from early 2020 [6,7]. The mutations include a deletion ( $\Delta 246-252)$ in the N-terminal domain (NTD), G75V and T76I in the NTD, L452Q and F490S in the receptor-binding motif, and $\mathrm{T} 859 \mathrm{~N}$ in the peptide between the fusion peptide and heptad repeat 1 (HR1) (Fig 1). With respect to the original strains and other variants, the 7-amino acid deletion and the L452Q and F490S mutations are novel.

\section{SPATIOTEMPORAL SPREAD OF THE LAMBDA VARIANT OF SARS-COV-2}

From data from dozens of countries, posted on the websites of national agencies of public health and professional

S1 S2

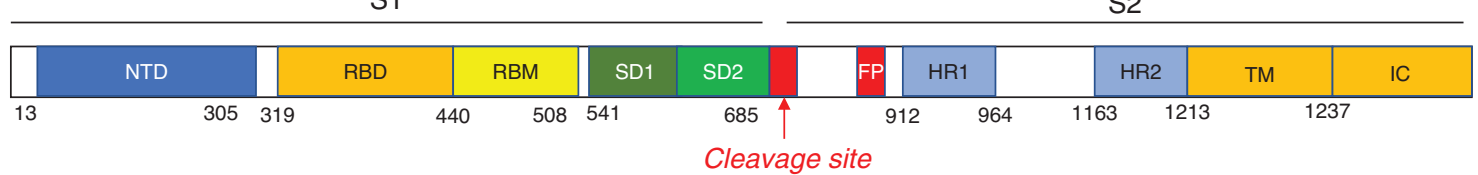

Cleavage site
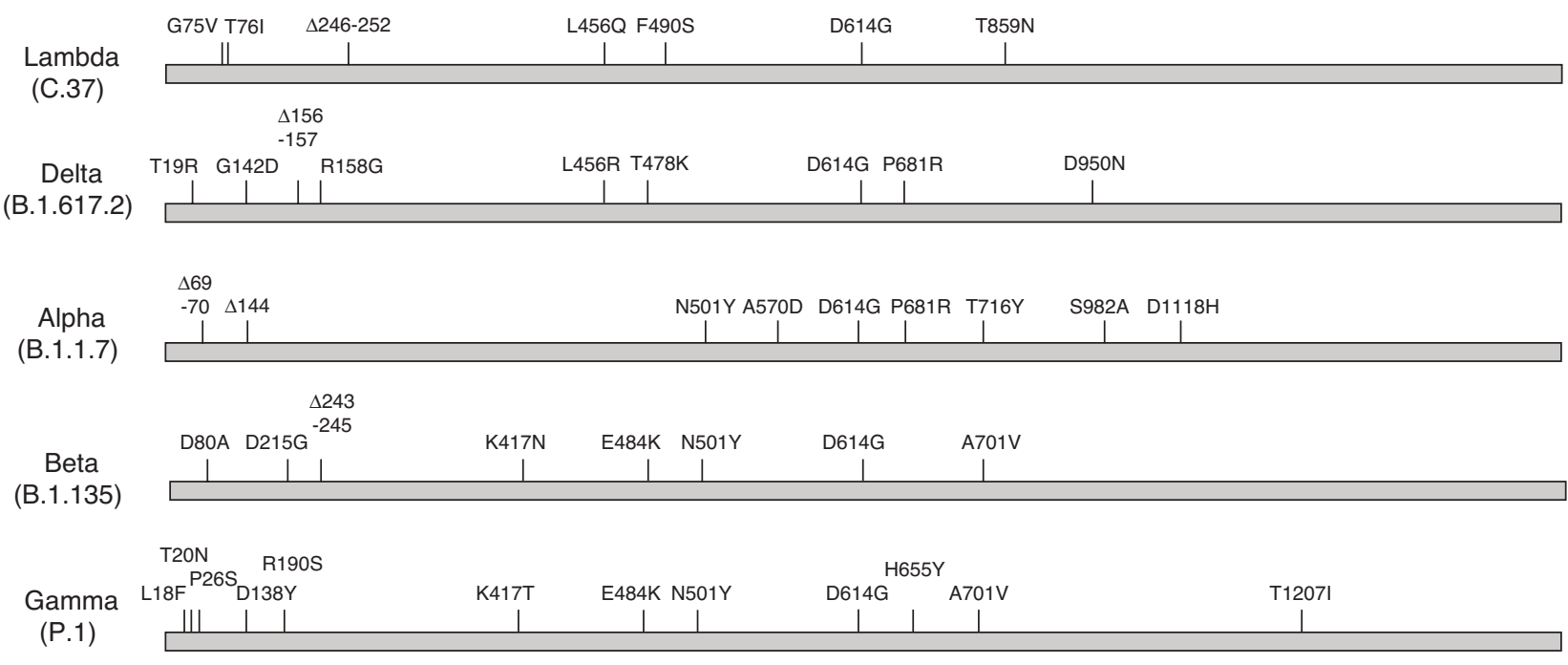

FIGURE 1 | Schematic structure of the spike protein of the Lambda (C.37) and Delta (B.1.617.2) variants. The mutations and deletions of the variants are indicated above. NTD: N-terminal domain; RBD: receptor-binding domain; RBM: receptor-binding motif; SD1: subdomain 1; SD2: subdomain 2; FP: fusion peptide; HR1: heptad repeat 1; HR2: heptad repeat 2; TM: transmembrane region; IC: intracellular domain. 
websites [8-10], the proportion of the Lambda variant of the SARS-CoV-2 sequence from the beginning of January to the end of July of 2021, and the spatiotemporal spread and distribution were analyzed. Until the end of July, seven countries from South America (Argentina, Brazil, Chile, Colombia, Ecuador, Peru and Uruguay) as well as 17 countries in the rest of the world (Australia, Belgium, Canada, Costa Rica, Denmark, France, Germany, Ireland, Italy, Japan, Mexico, the Netherlands, Spain, Sweden, Switzerland, the UK and the USA) had reported the exact proportions of the Lambda variant. The proportions of the Lambda variant in those countries at 13 time points from January 4 to July 26 are summarized in Table 1.

The Lambda variant emerged first in Peru in December 2020 and was responsible for only $2.3 \%$ of SARS-CoV-2 sequences at the beginning of January 2021. The proportion of the Lambda variant sequence increased rapidly and reached $27.36 \%$ at the end of January. After approximately 1.5-2 months, the Lambda variant became the predominant strain in Peru (81.82\% on March 22). In mid- to late May, the proportion of the Lambda variant sequence peaked (96.97\% on May 17), then slightly decreased, to approximately $80 \%$, in June and July.

Two neighboring counties, Argentina (2.78\%) and Chile (3.75\%), reported the proportions of the Lambda variant at the end of January. Unlike that in Peru, the spread of the Lambda variant in those two countries was slow. The Lambda variant in Argentina was maintained at a low level $(<5 \%)$ until the end of March, then increased to $17.17 \%$ on May 17 and peaked in June and July (26.67\% on June 28$)$. The proportion of the Lambda variant in Chile increased substantially from the beginning of March $16.67 \%$ on March 8), reached a peak in mid- to late April $(38.83 \%$ on April 19) and slightly decreased thereafter (27.3\% on July 26). Ecuador began to report the proportion of the Lambda SARS-CoV-2 sequence in mid- to late April $(17.54 \%$ on April 19). The proportion of the Lambda variant decreased in May and recovered to a level similar to that in April in

TABLE 1 | Temporal changes in the proportions of the Lambda variant in selected countries in South America and other regions.

\begin{tabular}{|c|c|c|c|c|c|c|c|c|c|c|c|c|c|c|}
\hline Region & Country & 4-Jan & 25-Jan & 8-Feb & $22-F e b$ & 8-Mar & 22-Mar & 5-Apr & 19-Apr & 3-May & 17-May & 14-Jun & 28-Jun & 26-Jul \\
\hline \multirow[t]{7}{*}{ S. America } & Argentina & 0 & 2.78 & 5.56 & 2.63 & 0 & 2.86 & 8.63 & 8.89 & 12.85 & 17.17 & 26.67 & 26.67 & ND \\
\hline & Brazil & 0 & 0 & 0 & 0 & 0.14 & 0 & 0 & 0 & 0 & 0 & 0.13 & 0.08 & 0 \\
\hline & Chile & 0 & 3.75 & 3.64 & 7.53 & 16.67 & 21.48 & 35.55 & 38.83 & 29.37 & 36.14 & 22.92 & 21.64 & 27.3 \\
\hline & Colombia & 0 & 0 & 0 & 0 & 0 & 1.31 & 0 & 2.79 & 0.75 & 2.63 & 1.2 & ND & ND \\
\hline & Ecuador & ND & ND & ND & ND & ND & ND & ND & 17.54 & 7.69 & 7.91 & 13.68 & 17.36 & 16.22 \\
\hline & Peru & 2.3 & 27.36 & 22.45 & 39.58 & 64.1 & 81.82 & 81.73 & 91.22 & 95.95 & 96.97 & 81.18 & 81.18 & 80.0 \\
\hline & Uruguay & ND & ND & ND & 0 & 0 & 0 & 0 & 0.75 & 0.75 & ND & ND & ND & ND \\
\hline \multirow[t]{17}{*}{ Others } & Australia & 0 & 0 & 0 & 0 & 0 & 0 & 0.86 & 0 & 0 & 0 & 0 & 0 & 0 \\
\hline & Belgium & 0 & 0 & 0 & 0 & 0 & 0 & 0 & 0 & 0 & 0 & 0 & 0.21 & 0.33 \\
\hline & Canada & 0 & 0 & 0 & 0.03 & 0.03 & 0.02 & 0.07 & 0.05 & 0.06 & 0.12 & 0 & 0.05 & 0 \\
\hline & Costa Rica & 0 & ND & ND & 0 & 0 & 0 & 0 & 0 & 0 & ND & 0 & 1.96 & 3.23 \\
\hline & France & 0 & 0 & 0 & 0 & 0 & 0 & 0 & 0 & 0 & 0.07 & 0.31 & 0.46 & 0.3 \\
\hline & Denmark & 0 & 0 & 0 & 0 & 0 & 0 & 0 & 0.01 & 0 & 0 & 0 & 0 & 0.02 \\
\hline & Germany & 0 & 0 & 0.03 & 0.03 & 0.09 & 0.02 & 0.04 & 0.06 & 0.05 & 0.06 & 0.28 & 0.04 & 0.05 \\
\hline & Ireland & 0 & 0 & 0 & 0 & 0 & 0 & 0 & 0 & 0 & 0 & 0 & 0 & 0.08 \\
\hline & Italy & 0 & 0 & 0 & 0 & 0 & 0 & 0 & 0 & 0 & 0.01 & 0.34 & 0.05 & 0 \\
\hline & Japan & 0 & 0 & 0 & 0 & 0 & 0 & 0 & 0 & 0 & 0 & 0 & 0 & 0.76 \\
\hline & Mexico & 0 & 0 & 0 & 0 & 0 & 0 & 0 & 0.4 & 1.84 & 0.9 & 1.55 & 2.63 & 0.2 \\
\hline & Netherlands & 0 & 0 & 0 & 0 & 0 & 0 & 0 & 0 & 0 & 0 & 0 & 0.06 & 0 \\
\hline & Spain & 0 & 0 & 0 & 0 & 0 & 0.04 & 0.18 & 0.24 & 0.3 & 0.33 & 0.93 & 1.01 & 0.06 \\
\hline & Sweden & 0 & 0 & 0 & 0 & 0 & 0 & 0 & 0 & 0 & 0 & 0 & 0 & 0.04 \\
\hline & Switzerland & 0 & 0 & 0 & 0 & 0 & 0 & 0 & 0 & 0 & 0 & 0 & 0.63 & 0.3 \\
\hline & UK & 0 & 0 & 0 & 0 & 0.01 & 0 & 0 & 0 & 0 & 0.01 & 0 & 0 & 0 \\
\hline & USA & 0 & 0 & 0.02 & 0.04 & 0.05 & 0.11 & 0.14 & 0.19 & 0.24 & 0.24 & 0.3 & 0.29 & 0.04 \\
\hline
\end{tabular}


June and July (16.22\% on July 28 ). The other three countries (Brazil, Colombia and Uruguay) also reported the percentages of the Lambda variant at later times, i.e., $0.14 \%$ on March 8 in Brazil, 1.31\% on March 22 in Colombia and 0.75\% on April 19 in Uruguay. Meanwhile, the prevalence of the Lambda variant in those three countries remained very low. The Gamma variant was the predominant strain in many countries in South America besides Peru.

The spread of the Lambda variant to the rest of world occurred slowly, although it was identified in the USA and Germany much earlier (in the beginning of February). Afterward, more European countries, Central and North American countries, and Australia and Japan reported the Lambda variant. However, the Lambda variant in all analyzed countries was maintained at very low proportions (Table 1). Mexico has continually reported the Lambda variant since April 19, but the percentage of this variant was lower than 3\%. Costa Rica has reported the Lambda variant since June 28, but the highest percentage was 3.23\%. The percentage of the Lambda variant in the other countries was below $1 \%$. Further analysis of the other countries, according to available data of the proportions of SARS-CoV-2 sequences, did not indicate the presence of the Lambda variant. These regions included China, India, Indonesia, Pakistan, Singapore, South Korea, Sri Lanka and Thailand in Asia; Austria, Poland, Norway, Romania and Turkey in Europe; Kenya, Nigeria and South Africa in Africa; and New Zealand in Oceania.

\section{ASSOCIATION OF THE PROPORTION OF THE LAMBDA VARIANT WITH OTHER EPIDEMIOLOGICAL INDEXES}

To explore the possible association of changes in the proportion of the Lambda variant with other epidemiological elements, we examined the temporal data of new confirmed cases per million, new deaths per million, R0 values, case fatality rate (CFR), tests per 1000, and percentages vaccinated and fully vaccinated (two doses) for populations in Argentina, Chile and Peru. Data on further circulation of the Lambda variant, observed in September, were separately collected as 7-day rolling averages [9]. As shown in Fig 2, the new cases and new deaths per million in Peru markedly increased from the beginning of January 2021, peaked in February and May, and clearly declined from June, together with the increase in the proportion of the Lambda variant sequence. The values in Argentina began to increase in late March and were maintained at high levels from late April to late June, whereas those in Chile began to increase in early March and remained high from late March to early July. Additionally, the numbers of new cases per million in Argentina and Chile were higher than those in Peru, whereas the numbers of new deaths per million were lower.

The CFR in these three countries did not substantially change during January to July of 2021 (Fig 3, orange curve). A clearly higher CFR (7.07-9.95) in Peru was observed, which declined to $<5$ in late June. The CFRs in Argentina
(1.15-2.24) and Chile (1.47-4.1) remained markedly low during this period. The $\mathrm{R} 0$ values during this period in the three countries also did not substantially change and remained near 1.0 (Fig 3, green curve). The numbers of tests per 1000 in the three countries increased from January to July (blue curve). Notably, the number of tests per 1000 in Peru at all time points was lowest, whereas that in Chile was highest. The percentages of vaccinated (one dose, gray area) and fully vaccinated (two doses, yellow area) people also increased over time but displayed different patterns in the three countries. The percentages of people receiving one dose and two doses in Chile at each time point were much higher than those in Argentina and Peru, which reached $72 \%$ and $64 \%$, respectively, on July 28 . Peru had the lowest percentage of the population vaccinated $(24 \%)$ and fully vaccinated (14\%), whereas Argentina had moderate vaccination coverage $(53 \%)$ and full vaccination $(13 \%)$.

From data obtained from 13 time points from the beginning of January to the end of July in 2021, the possible associations between with several epidemiological indexes and the CFR, new deaths per million (new deaths) and new confirmed cases per million (new cases) in Peru, Argentina and Chile at each time point were statistically analyzed in SPSS 22.0 software. The results of multivariate linear regression analysis are summarized in Table 2. For Peru, the alterations in the proportion of the Lambda variant, $\mathrm{R} 0$ values, test ratio (tests per 1000), and percentages vaccinated and fully vaccinated were not significantly associated with the CFR, new deaths and new cases. For Argentina, the alterations in the proportion of the Lambda variant and $\mathrm{R} 0$ value were significantly associated with new deaths, whereas the percentage vaccinated and the test ratio were significantly associated with the CFR. In Chile, the percentage vaccinated and the test ratios were significantly associated with new cases, whereas the percentage vaccinated was associated with new deaths.

\section{POTENTIAL EFFECTS ON TRANSMISSIBILITY, CLINICAL SEVERITY AND IMMUNE ESCAPE}

\section{Transmissibility}

The rapid spread of the Lambda variant was observed in Peru from January to May.This variant became the majority variant $(>50 \%)$ in early March and the predominant variant $(>80 \%)$ in late March $[9,11]$. The rapid increase in the Lambda lineage in the first quarter of 2021 in Peru was also reported in an analysis based on a genomic surveillance program [12]. A smaller increase in the circulation of the Lambda variant was also observed in neighboring countries, including Chile, Argentina and Ecuador, several months later, but the variant had not reached predominance by the end of July. Moreover, the circulation of the Lambda variant in Brazil and other South American countries remained at a very low level. A study using Bayesian skyline plot analysis has shown that the outbreak of the Lambda variant in South America was associated with the emergence of the novel deletion ( $\Delta 246-253)$ [7]. Although the Lambda strain 

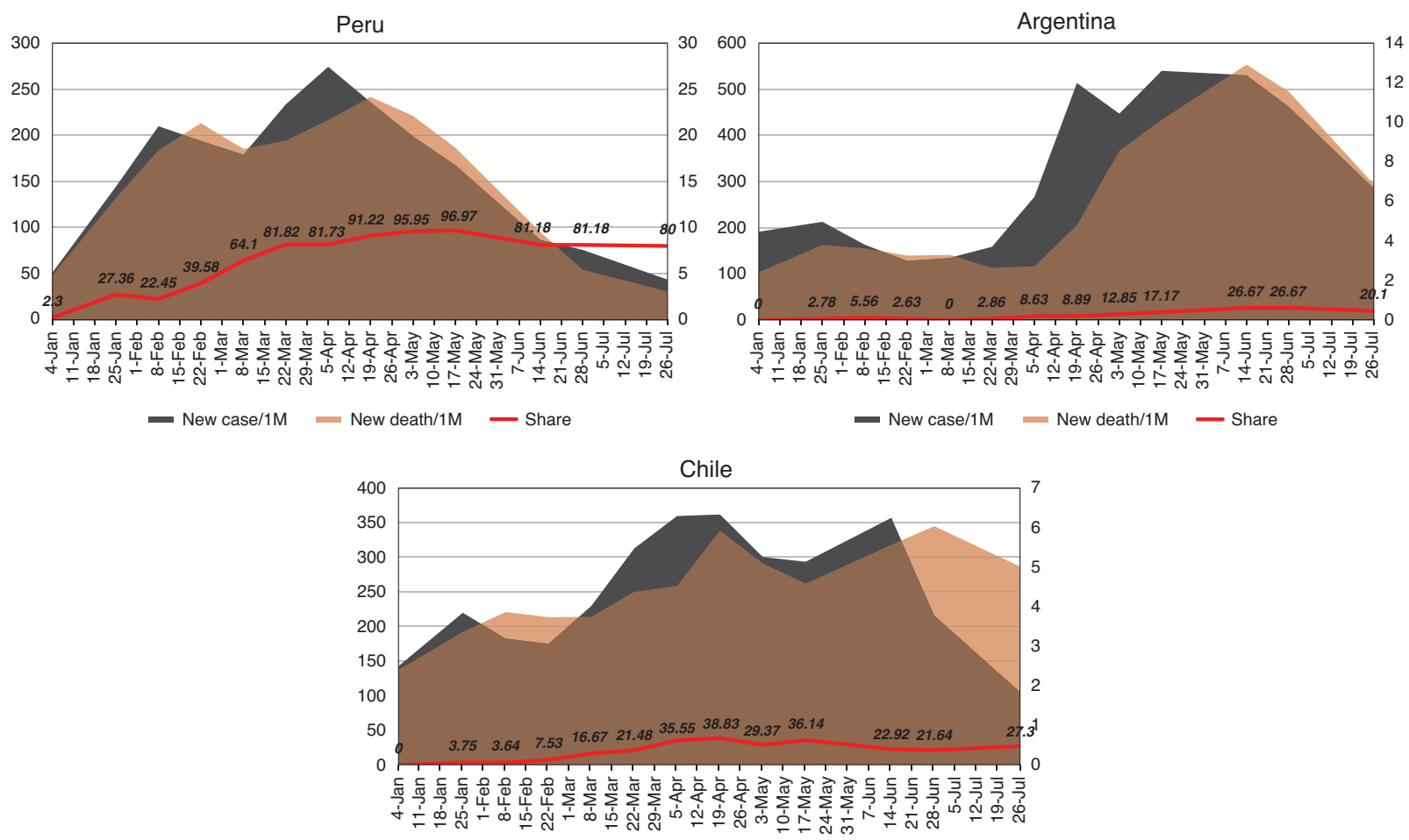

- New case/1M - New death/1M Share

FIGURE 2 | Alterations in the proportions of the Lambda variant, the new confirmed cases per million and the new deaths per million in Peru, Argentina and Chile from January 4 to July 26, 2021. Gray area: new cases per million; brown area: new deaths per million; red line: proportion of the Lambda variant. The data on new cases per million are shown on the left $Y$ axis. The data on new deaths and the proportion of the Lambda variant are shown on the right $Y$ axis.
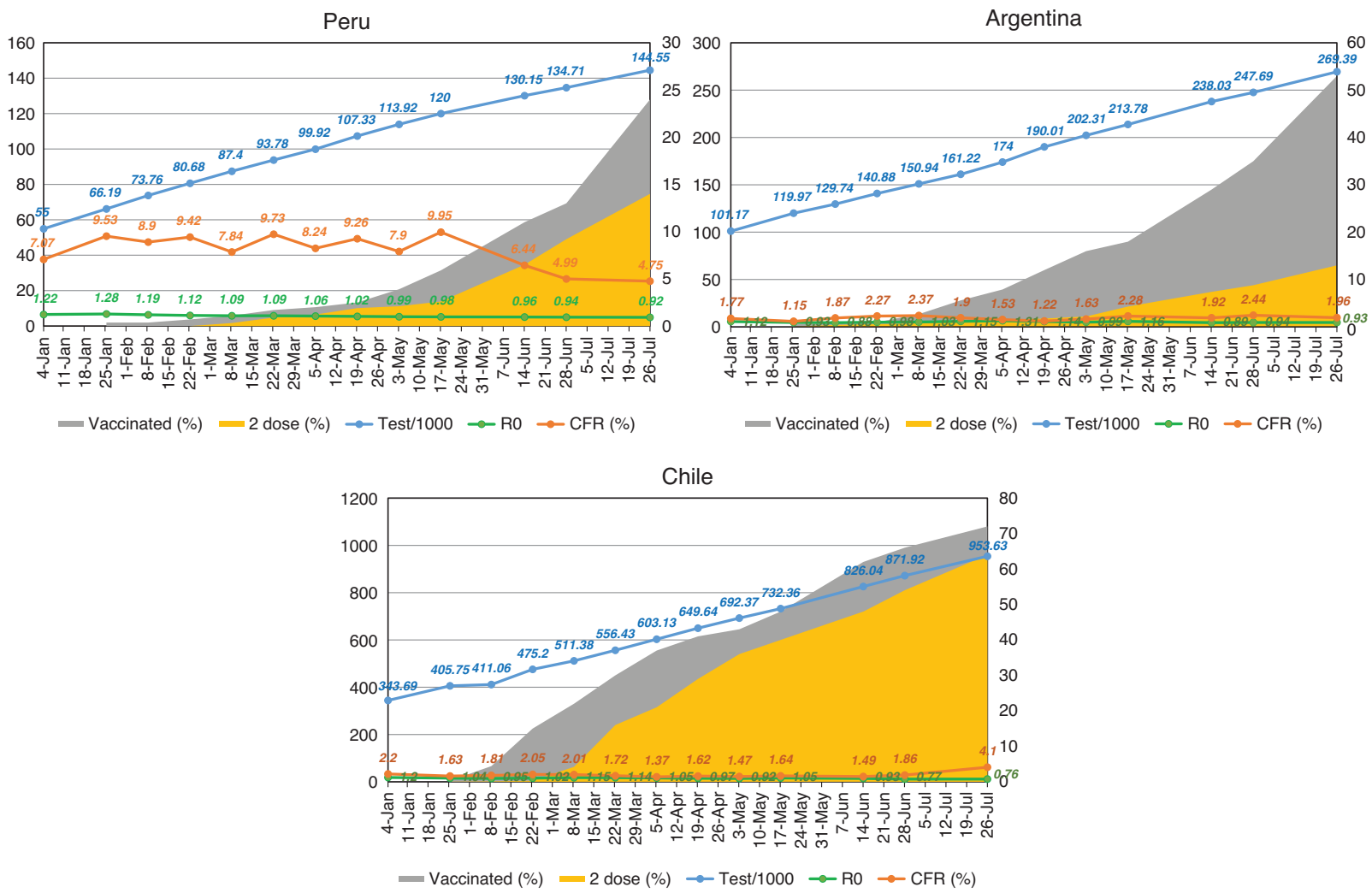

FIGURE 3 | Alterations in CFR (\%), R0 value, tests per 1000, and percentages vaccinated and fully vaccinated (two doses) in Peru, Argentina and Chile from January 4 to July 26, 2021. Gray area: vaccinated (\%); yellow area: fully vaccinated (\%); blue line: tests per 1000; orange line: CFR; green line: RO. The data of tests per 1000 are shown on the left $Y$ axis. The data on vaccinated (\%), fully vaccinated (\%), CFR and RO are shown on the right $Y$ axis. 
TABLE 2 | Multivariate linear regression analysis of the Lambda variant with various epidemiological factors in Peru, Argentina and Chile.

\begin{tabular}{|c|c|c|c|c|c|c|c|c|c|}
\hline & \multicolumn{3}{|c|}{ CFR (P-value) } & \multicolumn{3}{|c|}{ New deaths (P-value) } & \multicolumn{3}{|c|}{ New cases (P-value) } \\
\hline & Peru & Argentina & Chile & Peru & Argentina & Chile & Peru & Argentina & Chile \\
\hline Proportion (Lambda variant) & 0.51 & 0.088 & 0.405 & 0.293 & 0.032 & 0.977 & 0.173 & 0.062 & 0.843 \\
\hline RO & 0.388 & 0.15 & 0.786 & 0.124 & 0.04 & 0.069 & 0.377 & 0.934 & 0.384 \\
\hline Vaccinated (\%) & 0.196 & 0.009 & 0.086 & 0.647 & 0.242 & 0.033 & 0.719 & 0.405 & 0.034 \\
\hline Two doses (\%) & 0.09 & 0.012 & 0.109 & 0.358 & 0.993 & 0.232 & 0.791 & 0.082 & 0.056 \\
\hline Tests per 1000 & 0.682 & 0.028 & 0.052 & 0.206 & 0.083 & 0.054 & 0.225 & 0.518 & 0.023 \\
\hline
\end{tabular}

was detected in the USA and several European countries in early 2021, its proportion in the rest of the world has remained at a very low level. The greater transmissibility of the Lambda variant continues to be monitored.

\section{Severity}

The clinical features of COVID-19 associated with the Lambda SARS-CoV-2 variant have not been well documented. Moreover, the potential differences in severity between the Lambda variant and other variants remain unclear, although great concern has been expressed in the media. Well-designed cohort studies, particularly in South America, are warranted. Several studies have already revealed evidence of elevated infectivity of the Lambda variant in cells. A two-fold increase in the infectivity on cultured cells has also been observed with pseudotyped lentivirus carrying the Lambda spike protein [6]. Another study has identified that, in the context of pseudotyped virus, the Lambda variant has significantly higher infectivity than the parental D614G strain, and has similar infectivity to those of the Delta and Epsilon variants [7]. On the basis of an HIV-based pseudotyped virus platform, the generated pseudotyped virus containing the spike protein from Lambda variant has been found to produce higher infectivity in cultured cells than viruses containing D614G mutant spike proteins and other variants, such as Alpha and Gamma [13].

The high CFR of COVID-19 in Peru since 2021, accompanied by the prevalence of the Lambda variant, has led to assumptions that this variant should strongly affect severe outcomes. However, the CFR of COVID-19 in Peru was quite stable from January to May, ranging from 7.07 to 9.95, and in January, a relatively low proportion of the Lambda lineage sequence was reported. In fact, the cumulative CFR of COVID-19 until December of 2020 in Peru had already exceeded 9.0\%, while other strains and variants (Gamma and Alpha) also circulated in Peru. The number of tests per 1000 has been remarkably low for Peru, as compared with Argentina and Chile, thus leading to the speculation that the number of daily new confirmed cases has probably been underestimated. The COVID-19 vaccine coverage in Peru was also lower than those in with Argentina and Chile, thus also potentially influencing critical disease outcomes. Additionally, the proportion of people older than 64 years in Peru (10.92\%) is lower than those in Argentina (17.52\%) and Chile (16.16\%). Whether infection with the Lambda variant causes more critical and fatal illness still lacks clear evidence.

\section{Immune escape}

Unlike other variants, the Lambda variant has limited data indicating its influence on immunity and vaccination performance. In vitro neutralization assays have verified clearly diminished neutralization for the Lambda variant, mostly on the basis of the pseudotyped virus. Diminished neutralizing activities of convalescent sera collected before the emergence of the variant, as well as sera elicited by vaccination with mRNA vaccines (BNT162b2 and mRNA-1273) and adenoviral vector based vaccine (Ad26.COV2.S), have been observed against the pseudotyped virus carrying the Lambda spike protein, particularly Ad26.COV2.S-elicited antibody [14]. Another study from the same group has verified that the average neutralizing titers of convalescent sera and vaccine-elicited antibodies against the pseudotyped virus with the Lambda spike show 2.3- to 3.3-fold decreases compared to neutralization of virus with the parental D614G spike, thus indicating that the currently used vaccines are protective against the Lambda variant [6]. Another study based on pseudotyped virus has revealed similar results, indicating that the neutralizing titer of inactivated vaccine elicited antibodies against the Lambda variant decreased 3.05-fold, in contrast to a 2.33-fold decrease against the Gamma variant and 2.03-fold decrease against the Alpha variant compared to the wild type (lineage A) [13]. A similar limited decrease has also been observed in neutralization assays using mRNA COVID-19 vaccine elicited sera against various wild viruses isolated from clinical specimens, showing a 4.6-fold decrease in geometric mean titer against the Lambda variant (C.37), and 4.2-, 3.8and 3.0-fold decreases against the Beta (B.1.351), Alpha (B.1.1.7+E484K) and Delta (B.1.617.2) variants, respectively compared to the wild type (lineage A) [15].

The vaccine effectiveness of currently used COVID-19 vaccines against the Lambda variant in the real world has not yet been comprehensively determined, including the relevant infection; transmission; symptomatic cases; hospitalization; and severe, critical and fatal illness. The high vaccination rates in Chile and Argentina may provide benefits, given the relatively low CFR and mortality of COVID-19 in general. However, the effectiveness of vaccines toward the Lambda variant is unclear. 


\section{CONCLUSION}

In summary, the Lambda variant spread very rapidly in Peru and has been the predominant SARS-CoV-2 strain since March of 2021. Neighboring countries, i.e., Chile, Argentina and Ecuador, have also shown some degree of circulation of the Lambda variant, which still has not reached predominance. The circulation of the Lambda variant in other countries in South America (e.g., Brazil and Colombia) and in other regions of the world remains very low, although this variant was identified a long time ago. The infectivity of the Lambda variant on cultured cells is elevated, but its effects on clinical severity remain poorly documented. The Lambda variant displays some resistance to the in vitro neutralization of convalescent sera and vaccine-elicited antibodies. However, its exact effects on vaccine effectiveness in the real world must be further explored. The currently authorized COVID-19 vaccines are believed to still provide efficient protection. More comprehensive and active surveillance of the Lambda variant worldwide is crucial.

\section{FUNDING}

This work was supported by grants from the National Natural Science Foundation of China (grant no. 81630062) and the State Key Laboratory for Infectious Disease Prevention and Control, China CDC (grant nos. 2019SKLID501, 2019SKLID603, 2019SKLID307 and 2019SKLID401).

\section{COMPETING INTERESTS}

Prof. Xiao-Ping Dong is co-Editor-in-Chief of Zoonoses.

\section{REFERENCES}

1. WHO. Tracking SARS-CoV-2 variants. 2021 [cited 2021 Aug 15]. Available from: https://www.who.int/en/activities/ tracking-SARS-CoV-2-variants/.

2. ECDC. SARS-CoV-2 variants of concern as of 5 August 2021. 2021 [cited 2021 Aug 14]. Available from: https://www.ecdc. europa.eu/sites/default/files/documents/Variants-page-changelog-05-08.pdf.

3. USCDC. SARS-CoV-2 Variant Classifications and Definitions 2021 [cited 2021 Aug 15]. Available from: https:// www.cdc.gov/coronavirus/2019-ncov/variants/variantinfo.html?CDC_AA_refVal=https\%3A\%2F\%2Fwww.
cdc.gov\%2Fcoronavirus\%2F2019-ncov\%2Fcasesupdates $\% 2$ Fvariant-surveillance $\% 2$ Fvariant-info.html.

4. European Centre for Disease Prevention and Control. Threat Assessment Brief: Emergence of SARS CoV 2 B.1.617 variants in India and situation in the EU/ EEA. 2021 [cited 2021 Aug 15]. Available from: https://www.ecdc.europa.eu/en/publications-data/ threat-assessment-emergence-sars-cov-2-b1617-variants.

5. Shi Q, Dong XP. Rapid global spread of the SARS-CoV-2 Delta (B.1.617.2) variant: spatiotemporal variation and public health impact. Zooneses. 2021;1:3.

6. Tada TZ, Dcosta H, Samanovic BM, Mulligan MI, Mulligan MJ Landau NR. SARS-CoV-2 lambda variant remains susceptible to neutralization by mRNA vaccine-elicited antibodies and convalescent serum. bioRxiv, 2021.

7. Kimura I, Kosugi Y, Wu J, Yamasoba D, Butlertanaka EP, Tanaka YL, et al. SARS-CoV-2 Lambda variant exhibits higher infectivity and immune resistance. bioRxiv, 2021.

8. Coronavirus Pandemic (COVID-19). 2021 [cited 2021 Aug 15]. Available from: https://ourworldindata.org/coronavirus.

9. 2021 [cited 2021 Aug 16]. Available from: https://ourworldindata.org/explorers/coronavirus-data-explorer? zoomToSelection=true\&time=latest\&pickerSort=desc\& pickerMetric=gdp_per_capita\&Interval=Cumulative\&Relative+ to+Population=true\&Align+outbreaks=false\&Metric=People+ vaccinated $+\% 28$ by+dose $\% 29]$.

10. TRACKING Follow global cases and trends. Updated daily. 2021 [cited 2021 Aug 15]. Available from: https://coronavirus jhu.edu/data\#charts.

11. Romero PE, Dávila-Barclay A, Salvatierra G, González L, Cuicapuza D, Solis L, et al. The emergence of SARS-COV-2 variant lambdA (C.37) in South America. medRxiv. 2021.

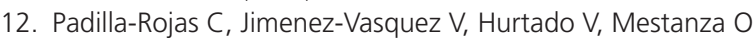
Molina IS, Barcena L, et al. Genomic analysis reveals a rapid spread and predominance of Lambda (C.37) SARS-COV-2 lineage in Peru despite circulation of variants of concern. J Med Virol. 2021.

13. Acevedo ML, Alonso-Palomares L, Bustamante A, Gaggero A, Paredes F, Cortés CP, et al. Infectivity and immune escape of the new SARS-CoV-2 variant of interest Lambda. medRxiv. 2021.

14. Tada T, Zhou H, Samanovic MI, Dcosta BM, Cornelius A Mulligan MJ, et al. Comparison of neutralizing antibody titers elicited by mRNA and adenoviral vector vaccine against SARS CoV-2 variants. bioRxiv. 2021

15. Carreño JM, Alshammary H, Singh G, Raskin A, Amanat $F$ Amoako $A$, et al. Reduced neutralizing activity of post-SARSCoV-2 vaccination serum against variants B.1.617.2, B.1.351, B.1.1.7+E484K and a sub-variant of C.37. medRxiv. 2021. 